\title{
A CLASS OF QTAG-MODULES AND RELATED CONCEPTS
}

\author{
RAFIQUDDIN $^{1, *}$, AYAZUL HASAN $^{2}$ \\ ${ }^{1}$ Department of Applied Mathematics, Faculty of Engineering and Technology, \\ Aligarh Muslim University, Aligarh-202002, India \\ ${ }^{2}$ College of Applied Industrial Technology, \\ Jazan University, Jazan, Kingdom of Saudi Arabia \\ *Corresponding author: rafiqamt786@rediffmail.com
}

Received Dec. 22, 2020

\begin{abstract}
Aвstract. The purpose of this paper is essentially to study $\alpha$-modules that depend on the notions of summability, purity, basic submodules, projectivity and injectivity. We call a $Q T A G$-module an $\alpha$-closed module if it is the maximal closed submodule of its closure in the $\alpha$-topology. It is found that an $\alpha$-closed $\alpha$-module is an $\alpha$-injective.

2010 Mathematics Subject Classification. 16K20.
\end{abstract}

Key words and phrases. $\alpha$-modules; $\alpha$-pure submodules; $\alpha$-basic submodules.

\section{Introduction and Background Material}

Modules are the natural generalizations of abelian groups. The results for abelian groups can be generalized for modules after imposing some conditions on modules/rings. In 1976 Singh [12] started the study of $T A G$-modules satisfying the following two conditions while the rings were associative with unity.

( $I)$ Every finitely generated submodule of any homomorphic image of $M$ is a direct sum of uniserial modules.

(II) Given any two uniserial submodules $U$ and $V$ of a homomorphic image of $M$, for any submodule $W$ of $U$, any non-zero homomorphism $f: W \rightarrow V$ can be extended to a homomorphism $g: U \rightarrow V$, provided the composition length $d(U / W) \leq d(V / f(W))$.

Later on Benabdallah, Singh, Khan etc. contributed a lot to the study of $T A G$-modules $[7,14]$. In 1987 Singh made an improvement and studied the modules satisfying only the condition $(I)$

DOI: $10.28924 /$ APJM/8-11

(92021 Asia Pacific Journal of Mathematics 
and called them $Q T A G$-modules. The study of $Q T A G$-modules and their structure began with work of Singh in [13]. This work, executed by many authors, clearly parallels the earlier work on torsion abelian groups. They studied different notions and structures on $Q T A G$-modules and developed the theory of these modules by introducing different notions and characterizing different submodules of $Q T A G$-modules. Yet there is much to explore.

All the rings $R$ considered here are associative with unity $(1 \neq 0)$ and modules $M$ are unital $Q T A G$-modules. A module $M$ in which the lattice of its submodule is totally ordered is called a serial module; in addition, if it has finite composition length, it is called a uniserial module. An element $x \in M$ is uniform, if $x R$ is a non-zero uniform (hence uniserial) module, and for any $R$-module $M$ with a unique decomposition series, $d(M)$ denotes its decomposition length. For a uniform element $x \in M, e(x)=d(x R)$ and $H_{M}(x)=$ $\sup \left\{d\left(\frac{y R}{x R}\right) \mid y \in M, x \in y R\right.$ and $y$ uniform $\}$ are the exponent and height of $x$ in $M$, respectively. $H_{k}(M)$ denotes the submodule of $M$ generated by the elements of height at least $k$ and $H^{k}(M)$ is the submodule of $M$ generated by the elements of exponents at most $k$. Let us denote by $M^{1}$, the submodule of $M$, containing elements of infinite height. The module $M$ is $h$-divisible if $M=M^{1}=\bigcap_{k=0}^{\infty} H_{k}(M)$. The module $M$ is $h$-reduced if it does not contain any $h$-divisible submodule. In other words, it is free from the elements of infinite height. The module $M$ is said to be bounded, if there exists an integer $n$ such that $H_{M}(x) \leq n$ for every uniform element $x \in M$.

The sum of all simple submodules of $M$ is called the socle of $M$, denoted by $\operatorname{Soc}(M)$ and a submodule $S$ of $\operatorname{Soc}(M)$ is called a subsocle of $M$. The cardinality of the minimal generating set of $M$ is denoted by $g(M)$. For all ordinals $\alpha, f_{M}(\alpha)$ is the $\alpha^{t h}-U l m$ invariant of $M$ and it is equal to $g\left(\operatorname{Soc}\left(H_{\alpha}(M)\right) / \operatorname{Soc}\left(H_{\alpha+1}(M)\right)\right)$.

A submodule $N$ of $M$ is $h$-pure in $M$ if $N \cap H_{k}(M)=H_{k}(N)$, for every integer $k \geq 0$. For an ordinal $\alpha$, a submodule $N \subseteq M$ is an $\alpha$-high submodule of $M$ if $N$ is maximal among the submodules of $M$ that intersect $H_{\alpha}(M)$ trivially.

For an ordinal $\alpha$, a submodule $N$ of $M$ is said to be an $\alpha$-pure, if $H_{\beta}(M) \cap N=H_{\beta}(N)$ for all $\beta \leq \alpha$ and a submodule $N$ of $M$ is said to be isotype in $M$, if it is $\alpha$-pure for every ordinal $\alpha$ [6]. A submodule $B \subseteq M$ is a basic submodule [10] of $M$, if $B$ is $h$-pure in $M, B=\oplus B_{i}$, where each $B_{i}$ is the direct sum of uniserial modules of length $i$ and $M / B$ is $h$-divisible. 
Imitating [4], the submodules $H_{k}(M), k \geq 0$ form a neighborhood system of zero, thus a topology known as $h$-topology arises. Closed modules are also closed with respect to this topology. Thus, the closure of $N \subseteq M$ is defined as $\bar{N}=\bigcap_{k=0}^{\infty}\left(N+H_{k}(M)\right)$. Therefore the submodule $N \subseteq M$ is closed with respect to $h$-topology if $\bar{N}=N$.

An $h$-reduced $Q T A G$-module $M$ is summable [11] if $\operatorname{Soc}(M)=\oplus_{\beta<\alpha} S_{\beta}$, where $S_{\beta}$ is the set of all elements of $H_{\beta}(M)$ which are not in $H_{\beta+1}(M)$, where $\alpha$ is the length of $M$. Moreover, $M$ is called totally projective [3], if $H_{\alpha}\left(\operatorname{Ext}\left(M / H_{\alpha}(M), M^{\prime}\right)\right)=0$ for all ordinal $\alpha$ and $Q T A G$ modules $M^{\prime}$.

It is interesting to note that almost all the results which hold for $T A G$-modules are also valid for $Q T A G$-modules [6]. Many results of this paper are the generalization of [5]. Our notations and terminology generally agree with those in [8] and [9].

\section{Chief Results}

For facilitating the exposition and for the convenience of the readers, we recall the definition of $\alpha$-modules from [2].

Definition 2.1. Let $\alpha$ denote the class of all $Q T A G$-modules $M$ such that $M / H_{\beta}(M)$ is totally projective for all ordinals $\beta<\alpha$, a limit ordinal. These modules are called $\alpha$-modules.

To develop the study, we need to prove some results, and we start with the following.

Proposition 2.1. If $N$ is an $\alpha$-pure submodule of an $\alpha$-module $M$, then $N$ is itself an $\alpha$-module.

Proof. We actually only need that $N \cap H_{\gamma}(M)=H_{\gamma}(N)$ for all $\gamma<\alpha$. For then it is a simple calculation to show that $N+H_{\beta}(M) / H_{\beta}(M)$ is isotype in $M / H_{\beta}(M)$ for each $\beta<\alpha$. And therefore, $N+H_{\beta}(M) / H_{\beta}(M) \cong N / H_{\beta}(N)$ is totally projective for all $\beta<\alpha$.

As generalized the notion of a basic submodule in [2], by defining $B$ to be an $\alpha$-basic submodule of an $\alpha$-module $M$ if $B$ is totally projective of length at most $\alpha, B$ is $\alpha$-pure submodule of $M$, and $M / B$ is $h$-divisible.

In order to establish the existence of $\alpha$-basic submodules we require the following notion for technical convenience.

Definition 2.2. Let $\alpha$ be a limit ordinal and $M$ a QT AG-module. An $\alpha$-high tower of $M$ is a wellordered ascending chain $\left\{M_{\beta}\right\}_{\beta<\alpha}$ of submodules of $M$ such that, for each $\beta, M_{\beta}$ is a $\beta$-high submodule of $M$. 
Now we need to prove the following lemma.

Lemma 2.1. Let $\alpha$ be a limit ordinal and $\left\{M_{\beta}\right\}_{\beta<\alpha}$ an $\alpha$-high tower of a $Q T A G$-module $M$. If each $M_{\beta}$ is summable, then $N=\bigcup_{\beta<\alpha} M_{\beta}$ is summable.

Proof. As $\alpha$ is a limit ordinal, we may choose a strictly increasing sequence $\beta_{1}<\beta_{2}<\cdots<$ $\beta_{n}<\ldots$ of ordinals having $\alpha$ as its limit. Then $N=\bigcup_{n<\omega} M_{\beta_{n}}$. Set $T_{0}=\operatorname{Soc}\left(M_{\beta_{1}}\right)$ and, for $n>1$, let $T_{n}$ be such that $\operatorname{Soc}\left(H_{\beta_{n}}(M)\right)=T_{n} \oplus \operatorname{Soc}\left(H_{\beta_{n+1}}(M)\right)$ with $T_{n} \subseteq M_{\beta_{n+1}}$. Then we have a direct-sum decomposition $\operatorname{Soc}(N)=\bigoplus_{n<\omega} T_{n}$ which is normal in the sense that $H_{M}\left(t_{1}+\cdots+t_{n}\right)=\min \left[H_{M}\left(t_{1}\right), \ldots, H_{M}\left(t_{n}\right)\right]$ provided $t_{i} \in T_{i}$ for $i=1, \ldots, n$. Now each $M_{\beta}$ is isotype, summable, and of countable length. Therefore, each subsocle of $M_{\beta}$ is a summable subsocle of $M$. In particular, each $T_{n}$ is a summable subsocle of $M$. Since the decomposition $\operatorname{Soc}(N)=\bigoplus_{n<\omega} T_{n}$ is normal, it follows that $\operatorname{Soc}(N)$ is a summable subsocle of $M$. Since each $M_{\beta}$ is isotype, $N$ is itself an isotype submodule of $M$ and consequently $N$ is summable.

We continue the study with the following corollary.

Corollary 2.1. Let $\alpha$ be a limit ordinal and $\left\{M_{\beta}\right\}_{\beta<\alpha}$ an $\alpha$-high tower of a $Q T A G$-module $M$, where each $M_{\beta}$ is totally projective, then $N=\bigcup_{\beta<\alpha} M_{\beta}$ is totally projective of length at most $\alpha$.

Proof. As noted above, $N$ is an isotype submodule of $M$ and clearly $N$ has a length at most $\alpha$. Thus $M_{\beta}$ is also a $\beta$-high submodule of $N$ for each $\beta<\alpha$. Since $N$ is summable by Lemma 2.1 implies that $N$ is totally projective.

Now we prove the following.

Theorem 2.1. Let $M$ be a $Q T A G$-module. Then $M$ contains an $\alpha$-basic submodule if and only if $M$ is an $\alpha$-module.

Proof. If $B$ is an $\alpha$-pure submodule of $M$ and if $M / B$ is $h$-divisible, then it follows that $M / H_{\beta}(M) \cong B / H_{\beta}(B)$ for all $\beta<\alpha$. Consequently, only $\alpha$-modules can have $\alpha$-basic submodules (see [2]). Suppose now that $M$ is an $\alpha$-module and select an $\alpha$-high tower $\left\{M_{\beta}\right\}_{\beta<\alpha}$. Now $M_{\beta} \cong M_{\beta}+H_{\beta}(M) / H_{\beta}(M)$, and since $M_{\beta}$ is isotype in $M, M_{\beta}+H_{\beta}(M) / H_{\beta}(M)$ is isotype in $M / H_{\beta}(M)$. By Corollary 2.1, $B=\bigcup_{\beta<\alpha} M_{\beta}$ is totally projective. It is easily seen that $\operatorname{Soc}(M) \subseteq \operatorname{Soc}(B)+H_{\beta}(M)$ for each $\beta<\alpha$, and therefore $B$ is $\alpha$-pure in $M$. Moreover, $B \cap H_{1}(M)=H_{1}(B)$ and $\operatorname{Soc}(M) \subseteq \operatorname{Soc}(B)+H_{\beta}(M)$ for $\beta<\omega$ imply that $M / B$ is $h$-divisible. Thus, $B$ is the required $\alpha$-basic submodule of $M$. 
Lemma 2.2. Suppose $N$ is an isotype submodule of a $Q T A G$-module $M$ and that $\left\{N_{\beta}\right\}_{\beta<\alpha}$ is an $\alpha$-high tower of $N$, then there exists an $\alpha$-high tower $\left\{M_{\beta}\right\}_{\beta<\alpha}$ of $M$ such that, for each $\beta, N_{\beta} \subseteq M_{\beta}$ and $N_{\beta}=N \cap M_{\beta}$.

Proof. Let us first note that $N_{\beta}=N \cap M_{\beta}$ is a consequence of $N_{\beta} \subseteq M_{\beta}$. Indeed, $N_{\beta} \subseteq M_{\beta}$ implies $N_{\beta} \subseteq N \cap M_{\beta}$ and $\left(N \cap M_{\beta}\right) \cap H_{\beta}(N)=\left(N \cap M_{\beta}\right) \cap H_{\beta}(M)=0$. The maximality of a $\beta$-high submodule then yields the equality. Assume now that $\beta<\alpha$ and that for each $\gamma<\beta$ we have a $\gamma$-high submodule $M_{\gamma}$ of $M$ such that $N_{\gamma} \subseteq M_{\gamma}$ and $M_{\eta} \subseteq M_{\gamma}$ for all $\eta<\gamma$. In order to be able to choose the desired $M_{\beta}$, it suffices to show that $\left(N_{\beta}+\cup_{\gamma<\beta} M_{\gamma}\right) \cap \operatorname{Soc}\left(H_{\beta}(M)\right)=0$. Suppose $x+y \in \operatorname{Soc}\left(H_{\beta}(M)\right)$ where $x \in N_{\beta}$ and $y \in M_{\gamma}$ for some $\gamma<\beta$. Then $H\left(x^{\prime}\right)=$ $-H\left(y^{\prime}\right) \in H_{1}(M) \cap N \cap M_{\gamma}=H_{1}(M) \cap N_{\gamma}=H_{1}\left(N_{\gamma}\right)$, where $d\left(\frac{x R}{x^{\prime} R}\right)=d\left(\frac{y R}{y^{\prime} R}\right)=1$, and hence there is $u \in N_{\gamma}$ such that $x-u \in \operatorname{Soc}(N)=\operatorname{Soc}\left(N_{\gamma}\right) \oplus \operatorname{Soc}\left(H_{\gamma}(N)\right)$. Thus we can write $x=u+v+z$ where $v \in \operatorname{Soc}\left(N_{\gamma}\right)$ and $z \in \operatorname{Soc}\left(H_{\gamma}(N)\right)$. Then $u+v+y=x+y-z \in$ $H_{\gamma}(M) \cap M_{\gamma}=0$ and $x+y=z \in N$. Therefore $y \in N \cap M_{\gamma}=N_{\gamma} \subseteq N_{\beta}$ and, consequently, $x+y \in N_{\beta} \cap H_{\beta}(M)=N_{\beta} \cap H_{\beta}(N)=0$ as desired.

Lemma 2.3. Let $M$ be a totally projective $Q T A G$-module such that $M=\bigcup_{\beta<\alpha} M_{\beta}$ where $\left\{M_{\beta}\right\}_{\beta<\alpha}$ is an $\alpha$-high tower. If $N$ is an $\alpha$-pure submodule of $M$ such that for each $\beta, N \cap M_{\beta}$ is a $\beta$-high submodule of $N$, then $N$ is a direct summand of $M$.

Proof. We need only show that $M / N$ is totally projective having length at most $\alpha$. Since $N \cap M_{\beta}$ is $(\beta+1)$-pure in $N$ and $N$ is $\alpha$-pure in $M, N \cap M_{\beta}$ is $(\beta+1)$-pure in $M$ and, a fortiori, $(\beta+1)$-pure in $M_{\beta}$. Since $M_{\beta}$ is totally projective, $M_{\beta}$ is $\beta$-projective. Therefore, there is direct decomposition $M_{\beta}=\left(N \cap M_{\beta}\right) \oplus K_{\beta}$ for each $\beta<\alpha$. Now $M / N=\bigcup_{\beta<\alpha} M_{\beta}+N / N$ and $M_{\beta}+N / N \cong M_{\beta} /\left(M_{\beta} \cap N\right) \cong K_{\beta}$ is totally projective for each $\beta$. By Corollary 2.1, it is enough to show that $M_{\beta}+N / N$ is a $\beta$-high submodule of $M / N$ whenever $\omega \leq \beta<\alpha$. Since $N$ is $\alpha$-pure in $M$, we have $\operatorname{Soc}\left(H_{\beta}(M / N)\right)=\operatorname{Soc}\left(H_{\beta}(M)\right)+N / N$ for $\beta<\alpha$ and it then easily follows that $\operatorname{Soc}(M / N)=\operatorname{Soc}\left(M_{\beta}+N / N\right) \oplus \operatorname{Soc}\left(H_{\beta}(M / N)\right)$. Because of this direct decomposition, it is enough to show that $M_{\beta}+N / N$ is an $h$-pure submodule of $M / N$ for $\beta \geq \omega$.

Now

$$
\begin{aligned}
\operatorname{Soc}\left(M_{\beta}+N\right) & =\operatorname{Soc}\left(K_{\beta} \oplus N\right) \\
& =\operatorname{Soc}\left(K_{\beta}\right) \oplus \operatorname{Soc}(N) \\
& =\operatorname{Soc}\left(K_{\beta}\right) \oplus \operatorname{Soc}\left(N \cap M_{\beta}\right) \oplus \operatorname{Soc}\left(H_{\beta}(N)\right) \\
& =\operatorname{Soc}\left(M_{\beta}\right) \oplus \operatorname{Soc}\left(H_{\beta}(N)\right) .
\end{aligned}
$$


If $\beta \geq \omega$ and if $x \in \operatorname{Soc}\left(M_{\beta}+N\right)$, then we can write $x=y+z$ where $y \in \operatorname{Soc}\left(M_{\beta}\right)$ and $z \in \operatorname{Soc}\left(H_{\beta}(N)\right) \subseteq H_{\omega}(N)$. If $x$ has finite height in $M$, then this height is just the height of $y$ in $M$ (= height of $y$ in $M_{\beta}$ ) and thus just the height of $x=y+z$ in $M_{\beta}+N$. On the other hand, if $x$ has infinite height in $M$, then $y$ has infinite height in $M_{\beta}$ and $x=y+z$ has infinite height in $M_{\beta}+N$, it follows that $M_{\beta}+N$ is an $h$-pure submodule of $M$. Thus $M_{\beta}+N / N$ is $h$-pure in $M / N$.

Proposition 2.2. Let $N$ be an $\alpha$-pure submodule of an $\alpha$-module $M$ such that $N$ is totally projective of length at most $\alpha$. Then there exists a submodule $K$ of $M$ such that $N \oplus K$ is an $\alpha$-basic submodule of $M$.

Proof. Since $N$ is totally projective of length $\leq \alpha, N$ is the union of an $\alpha$-high tower $\left\{N_{\beta}\right\}_{\beta<\alpha}$ of itself. By Lemma 2.2, there exists an $\alpha$-high tower $\left\{M_{\beta}\right\}_{\beta<\alpha}$ of $M$ such that $N_{\beta}=N \cap M_{\beta}$ for each $\beta$. Let $B=\bigcup_{\beta<\alpha} M_{\beta}$. By the proof of Theorem 2.1, $B$ is an $\alpha$-basic submodule of $M$. But $\left\{M_{\beta}\right\}_{\beta<\alpha}$ is also an $\alpha$-high tower of $B$, and by Lemma 2.3 we have the required direct decomposition $B=N \oplus K$.

Now we prove the following result.

Theorem 2.2. If $N$ is an $\alpha$-pure submodule of an $\alpha$-module $M$, then $M / N$ is an $\alpha$-module.

Proof. Let $B$ be an $\alpha$-basic submodule of $N$ and choose $K$ such that $B \oplus K$ is an $\alpha$-basic submodule of $M$. Now if $x \in \operatorname{Soc}(N \cap K)$, we can write for each $\beta<\alpha, x=y_{\beta}+z_{\beta}$, where $y_{\beta} \in \operatorname{Soc}(N)$ and $z_{\beta} \in H_{\beta}(N)$. Thus $-y_{\beta}+x \in H_{\beta}(B \oplus K)=H_{\beta}(B) \oplus H_{\beta}(K)$ and $x \in \bigcap_{\beta<\alpha} H_{\beta}(K)=H_{\alpha}(K)=0$. We then have a direct decomposition $N \oplus K$. If $H_{1}\left(a^{\prime}\right) \in N \oplus K$, then $H_{1}\left(a^{\prime}\right)=y+H_{1}\left(b^{\prime}\right)+c$, where $d\left(\frac{a R}{a^{\prime} R}\right)=d\left(\frac{b R}{b^{\prime} R}\right)=1, y \in B, b \in N$ and $c \in K$. Since $H_{1}(M) \cap(B \oplus K)=H_{1}(B \oplus K)$, we conclude that $H_{1}(M) \cap(N \oplus K)=H_{1}(N \oplus K)$. Now $\operatorname{Soc}(M) \subseteq \operatorname{Soc}(B \oplus K)+H_{\beta}(M) \subseteq \operatorname{Soc}(N \oplus K)+H_{\beta}(M)$ for all $\beta<\alpha$, and therefore $N \oplus K$ is an $\alpha$-pure submodule of $M$. Consequently, $N \oplus K / N$ is $\alpha$-pure in $M / N$. Also $N \oplus K / N \cong K$ and $(M / N) /(N \oplus K / N) \cong(M / B \oplus K) /[(N \oplus K) /(B \oplus K)]$ is $h$-divisible. We have constructed an $\alpha$-basic submodule of $M / N$ and we conclude that $M / N$ is indeed an $\alpha$-module.

As a consequence of the above theorem, we have the following striking analog of a familiar property of $h$-pure submodules.

Corollary 2.2. Let $N$ be a submodule of an $\alpha$-module $M$. Then $N$ is an $\alpha$-pure submodule of $M$ if and only if $N+H_{\beta}(M) / H_{\beta}(M)$ is a direct summand of $M / H_{\beta}(M)$ for all $\beta<\alpha$. 
Proof. $N+H_{\beta}(M) / H_{\beta}(M)$ being a direct summand of $M / H_{\beta}(M)$ implies that $N+H_{\beta}(M) / H_{\beta}(M)$ is $\beta$-pure in $M / H_{\beta}(M)$, which is equivalent to $N$ being $\beta$-pure in $M$. Since $\alpha$ is a limit ordinal, $N$ is $\alpha$-pure in $M$ if and only if $N$ is $\beta$-pure in $M$ for all $\beta<\alpha$.

Conversely, assume that $N$ is $\alpha$-pure in $M$. Then $M / N$ is an $\alpha$-module and therefore, for $\beta<\alpha$,

$$
(M / N) / H_{\beta}(M / N)=(M / N) /\left(H_{\beta}(M)+N / N\right) \cong\left(M / H_{\beta}(M)\right) /\left(N+H_{\beta}(M) / H_{\beta}(M)\right)
$$

is totally projective of length at most $\beta$. Since $N+H_{\beta}(M) / H_{\beta}(M)$ is $\beta$-pure in $M / H_{\beta}(M)$, $N+H_{\beta}(M) / H_{\beta}(M)$ is a direct summand of $M / H_{\beta}(M)$.

Proposition 2.3. If $N$ is an $\alpha$-pure submodule of an $\alpha$-module $M$, and if $H_{\beta}(N)$ is a direct summand of $H_{\beta}(M)$ for some $\beta<\alpha$, then $N$ is a direct summand of $M$.

Proof. Assuming the conditions of the Theorem 2.2, we have for some $\beta<\alpha$ :

(i) $(M / N) / H_{\beta}(M / N)$ is totally projective;

(ii) $N \cap H_{\beta}(M)=H_{\beta}(N)$;

(iii) $N+H_{\beta}(M) / H_{\beta}(M)$ is a direct summand of $M / H_{\beta}(M)$; and

(iv) $H_{\beta}(M)=H_{\beta}(N) \oplus K$.

It follows that $M=N \oplus L$ where $L \supseteq K$.

As a corollary, we have the following generalization of the well-known fact that bounded $h$-pure submodules are direct summands.

Corollary 2.3. If $N$ is an $\alpha$-pure submodule of an $\alpha$-module $M$ and if $H_{\beta}(N)=0$ for some $\beta<\alpha$, then $N$ is a direct summand of $M$.

As defined in [3], a $Q T A G$-module $M$ is fully transitive if for every pair of uniform elements $x, y \in M, H_{M}\left(x_{i}\right) \leq H_{M}\left(y_{i}\right)$ for all $i \geq 0$ implies that there exists an endomorphism of $M$ that maps $x$ onto $y$. Here $d\left(\frac{x R}{x_{i} R}\right)=d\left(\frac{y R}{y_{i} R}\right)=i$.

The next corollary tells us that $\alpha$-modules of length $\alpha$ are fully transitive (see [1]). This, of course, is merely a reflection of the fact that modules of length $\leq \alpha$ behave in the $\alpha$ context exactly as modules without elements of infinite height in the classical situations.

Corollary 2.4. If $M$ is an $\alpha$-module of length $\alpha$, then every finite subset of $M$ is contained in a countably generated direct summand. 
Proof. Let $S$ be a finite subset of $M$. Then $S \subseteq T$ for some countably generated, $\alpha$-pure submodule $T$ of $M$. We may assume that $T$ has length $\alpha$. Then $T$ is a direct sum of modules of length less than $\alpha$. Consequently, $T$ is contained in a direct summand $K$ of $T$ having length less than $\alpha$. By the preceding corollary, $K$ is a direct summand of $M$.

For a limit ordinal $\alpha$, an $\alpha$-module $M$ is an $\alpha$-projective if $H_{\alpha}\left(\operatorname{Ext}\left(M, M^{\prime}\right)\right)=0$ for all $\alpha$-modules $M^{\prime}$, that is, there exists a submodule $N$ bounded by $\alpha$ such that $M / N$ is totally projective, and an $\alpha$-module $M$ is an $\alpha$-injective if $H_{\alpha}\left(\operatorname{Ext}\left(M^{\prime}, M\right)\right)=0$ for all $\alpha$-modules $M^{\prime}$, that is, it is a direct summand of every $\alpha$-module in which it occurs as an $\alpha$-pure submodule.

To characterize the $\alpha$-injective modules we must generalize the notion of a closed module. Mimicking [2], for any $Q T A G$-module $M$, the submodules $\left\{H_{k}(M)\right\}_{k}, k=0,1,2, \ldots, \infty$ from a neighborhood system of zero, giving rise to $h$-topology. If $k$ is replaced by an arbitrary limit ordinal less than or equal to $\alpha$, then $h$-topology may be extended to $\alpha$-topology, and all the definitions and results which hold for $h$-topology may be extended for $\alpha$-topology. In $\alpha$-topology, for any submodule $N$ of $M$, the closure of $N$ as $\bigcap_{\beta<\alpha}\left(N+H_{\beta}(M)\right)$ denoted by $\bar{N}$.

Definition 2.3. We call a $Q T A G$-module an $\alpha$-closed module if it is the maximal closed submodule of its closure in the $\alpha$-topology.

With the help of the above discussion, we are able to infer the following.

Proposition 2.4. Let $M$ be an $\alpha$-closed $\alpha$-module. Then $M$ is an $\alpha$-injective.

Proof. We first show that $H_{\alpha}(\operatorname{Ext}(T, M))=0$ for all $\alpha$-modules $T$. Assume that $M$ is an $\alpha$-pure submodule of $M^{\prime}$ with $M^{\prime} / M \cong T$ for all $\alpha$-modules $M^{\prime}$. Since $\alpha$ is a limit ordinal, it follows that $M^{\prime}=H_{\beta}\left(M^{\prime}\right)+M$ for all $\beta<\alpha$. Therefore, if $y \in M^{\prime}$, we can find for each $\beta<\alpha$ a $x_{\beta} \in M$ such that $y-x_{\beta} \in H_{\beta}\left(M^{\prime}\right)$. Moreover, we can assume that the exponent of $x_{\beta}$ does not exceed that of $y$. Indeed, if $y$ has exponent $n$, then $H_{n}\left(x_{\beta}^{\prime}\right) \in H_{\beta+n}\left(M^{\prime}\right) \cap M=H_{\beta+n}(M)$, where $d\left(\frac{x_{\beta} R}{x_{\beta}^{\prime} R}\right)=n$ and $H_{n}\left(x_{\beta}^{\prime}\right)=H_{n}\left(z_{\beta}^{\prime}\right)$, where $d\left(\frac{x_{\beta} R}{x_{\beta}^{\prime} R}\right)=d\left(\frac{z_{\beta} R}{z_{\beta}^{\prime} R}\right)=n$ for some $z_{\beta} \in H_{\beta}(M)$. Then $\bar{x}_{\beta}=x_{\beta}-z_{\beta}$ has an exponent at most $n$ and $y-\bar{x}_{\beta} \in H_{\beta}\left(M^{\prime}\right)$. But $\left\{x_{\beta}: \beta<\alpha\right\}$ is a chain in $M$ with elements uniformly bounded in exponent and, therefore, converges to some $x \in M$. Hence $y-x \in \bigcap_{\beta<\alpha} H_{\beta}\left(M^{\prime}\right)=H_{\alpha}\left(M^{\prime}\right)$. We conclude that $M^{\prime}=M \oplus H_{\alpha}\left(M^{\prime}\right)$.

Now let $M^{\prime}$ be an arbitrary $\alpha$-module and let $B$ be an $\alpha$-basic submodule of $M^{\prime}$. We then have the exact sequence

$$
H_{\alpha}\left(\operatorname{Ext}\left(M^{\prime} / B, M\right)\right) \longrightarrow H_{\alpha}\left(\operatorname{Ext}\left(M^{\prime}, M\right)\right) \longrightarrow H_{\alpha}(\operatorname{Ext}(B, M)) .
$$


The left-hand term of the above sequence vanishes since $M^{\prime} / B$ is isomorphic to a direct sum of copies of $T$ and the right-hand term vanishes since $B$ is an $\alpha$-projective. Thus, $H_{\alpha}\left(\operatorname{Ext}\left(M^{\prime}, M\right)\right)=0$ and we conclude that $M$ is an $\alpha$-injective.

We can now show that there are enough $\alpha$-injective modules and that an $\alpha$-injective module is the sum of an $\alpha$-closed module and an $h$-divisible module.

Theorem 2.3. Let $M$ be an $\alpha$-module. Then $M$ is an $\alpha$-pure submodule of an $\alpha$-injective module and $M$ is an $\alpha$-injective module if and only if $M$ is the direct sum of an h-divisible module and an $\alpha$-closed $\alpha$-module.

Proof. It is evident from Proposition 2.4 that the direct sum of an $h$-divisible module and an $\alpha$-closed $\alpha$-module is necessarily an $\alpha$-injective. Next, we need the observation that every $\alpha$-module $M$ of length at most $\alpha$ can be imbedded as an $\alpha$-pure submodule of an $\alpha$-closed module $T_{M}(\alpha)$ such that $T_{M}(\alpha) / M$ is $h$-divisible. Indeed, $T_{M}(\alpha)$ may be taken as the maximal closed submodule of the closure of $M$ in the $\alpha$-topology. It follows, by the same reasoning as in the proof of Theorem 2.1, that $T_{M}(\alpha) / H_{\beta}\left(T_{M}(\alpha)\right) \cong M / H_{\beta}(M)$ for all $\beta<\alpha$, and therefore that $T_{M}(\alpha)$ is an $\alpha$-module.

Now let $M$ be an arbitrary $\alpha$-module. Let $D$ be a minimal $h$-divisible module containing $H_{\alpha}(M)$. Take $P$ to be the amalgamated sum of $M$ and $D$ over $H_{\alpha}(M)$. Then $P=M^{\prime} \oplus D$ where $M^{\prime} \cong M / H_{\alpha}(M)$ and $M^{\prime} \cap M$ is an $\alpha$-high submodule of $M$. Also, $P / M$ is $h$-divisible and $\operatorname{Soc}(P) \subseteq \operatorname{Soc}(M)+H_{\beta}(P)$ for all $\beta<\alpha$. It follows that $M$ is an $\alpha$-pure submodule of $P$. By the transitivity of $\alpha$-purity, $M$ is an $\alpha$-pure in the $\alpha$-injective $T_{M^{\prime}}(\alpha) \oplus D$.

Finally, assume that $M$ is itself an $\alpha$-injective and that we have it imbedded, as above, as an $\alpha$-pure submodule of $\bar{P}=T_{M^{\prime}}(\alpha) \oplus D$. Since $M$ is an $\alpha$-injective, $\bar{P}=M \oplus Q$ where $Q \cong \bar{P} / M$ is obviously $h$-divisible, since both $P / M$ and $\bar{P} / P$ are $h$-divisible. But then $Q \subseteq D$, and since $\operatorname{Soc}(D) \subseteq H_{\alpha}(M)$, we conclude that $Q=0$ and $M=T_{M^{\prime}}(\alpha) \oplus D$.

Now we are in a position to prove the following result.

Theorem 2.4. If $M$ and $M^{\prime}$ are $\alpha$-closed $\alpha$-modules with the same Ulm invariants, then $M \cong M^{\prime}$.

Proof. Take $B$ and $B^{\prime}$ to be $\alpha$-basic submodules of $M$ and $M^{\prime}$, respectively. It is easily seen that $B$ and $B^{\prime}$ have the same $U l m$ invariants as $M$ and $M^{\prime}$. Therefore, there is an isomorphism $f$ of $B$ onto $B^{\prime}$. Since $B$ is an $\alpha$-pure submodule of $M$, we have the exact sequence

$$
\operatorname{Hom}\left(M, M^{\prime}\right) \longrightarrow \operatorname{Hom}\left(B, M^{\prime}\right) \longrightarrow H_{\alpha}\left(\operatorname{Ext}\left(M / B, M^{\prime}\right)\right)=0
$$


Thus, there is a homomorphism $f^{\prime}: M \longrightarrow M^{\prime}$ that extends $f$. Let $x \in \operatorname{Ker} f^{\prime}$ and assume that $x \neq 0$. Then $x$ has some height $\beta<\alpha$ and we can write $x=y+z$ where $y \in B$ and $z \in H_{\beta+1}(M)$. But then $x$ has height $\beta$ and $f(y)=f^{\prime}(y)=-f^{\prime}(z)$ has height at least $\beta+1$. This, however, is a contradiction, since $f$ is an isomorphism of $B$ onto $B^{\prime}$ and $B^{\prime}$ is an isotype submodule of $M^{\prime}$. We conclude that $\operatorname{Ker} f^{\prime}=0$. Then $f^{\prime}(M) / B^{\prime}=f^{\prime}(M) / f^{\prime}(B) \cong M / B$ is $h$-divisible. Hence $f^{\prime}(M) / B^{\prime}$ is a direct summand of $M^{\prime} / B^{\prime}$, and since $B^{\prime}$ is an $\alpha$-pure submodule of $M^{\prime}$, it follows that $f^{\prime}(M)$ is an $\alpha$-pure submodule of $M^{\prime}$. Since $f^{\prime}(M) \cong M$ is an $\alpha$-injective, we have a direct decomposition $\left.M^{\prime}=f^{\prime}(M)\right) \oplus L$ where $L \cong M^{\prime} / f^{\prime}(M)$ is $h$-divisible. But $M^{\prime}$ is $h$-reduced and therefore $L=0$ and $f^{\prime}(M)=M^{\prime}$, that is, $f^{\prime}$ is an isomorphism of $M$ onto $M^{\prime}$.

\section{ACKNOWLEDGMENTS}

The authors express their appreciation to the referee and to the editor, for their valuable assistance in processing our work.

\section{REFERENCES}

[1] A. Hasan, On generalized submodules of QTAG-modules, Georgian Math. J. 23(2016), no. 2, 221-226.

[2] A. Mehdi, F. Sikander and S.A.R.K. Naji, Generalizations of basic and large submodules of QTAG-modules, Afr. Mat. 25(2014), no. 4, 975-986.

[3] A. Mehdi, M.Y. Abbasi and F. Mehdi, Nice decomposition series and rich modules, South East Asian J. Math. Math. Sci. 4(2005), no. 1, 1-6.

[4] A. Mehdi, S.A.R.K. Naji and A. Hasan, Small homomorphisms and large submodules of QTAG-modules, Sci. Ser. A. Math Sci. 23(2012), 19-24.

[5] C. Megibben, A Generalization of classical theory of primary abelian groups, Tohoku Math. J. 22(1970), 344-356.

[6] H. Mehran and S. Singh, On $\sigma$-pure submodules of QTAG-modules, Arch. Math. 46(1986), 501-510.

[7] K. Benabdallah and S. Singh, On torsion abelian groups like modules, Lecture Notes in Mathematics, Spriger Verlag, 1006(1983), 639-653.

[8] L. Fuchs, Infinite Abelian Groups, Vol. I, Academic Press, New York, 1970.

[9] L. Fuchs, Infinite Abelian Groups, Vol. II, Academic Press, New York, 1973.

[10] M.Z. Khan, $h$-divisible and basic submodules, Tamkang J. Math. 10(1979), no. 2, 197-203.

[11] S.A.R.K. Naji, A study of different structures in QTAG-modules, Ph.D. thesis, Aligarh Muslim University, 2010.

[12] S. Singh, Some decomposition theorems in abelian groups and their genesalizations, Ring Theory: Proceedings of Ohio University Conference, Marcel Dekker, New York 25, 183-189, (1976).

[13] S. Singh, Abelian groups like modules, Act. Math. Hung, 50(1987), 85-95. 
[14] S. Singh and M.Z. Khan, $T A G$-modules with complement submodules $h$-pure, Internat. J. Math. Math. Sci. 21(1998), no. 4, 801-814. 